\title{
Assessment of the quality of directly observed treatment short-course of tuberculosis in Bahir Dar city administration, North West Ethiopia
}

\author{
Mulatu Kassie ${ }^{1}$, Amanu Aragaw², Alemayehu Belay ${ }^{3}$ \\ ${ }^{1}$ Department of community health, Bahir Dar health Science College, Bahir Dar city, Ethiopia \\ ${ }^{2}$ Academic Vice dean Bahir Dar health science college, Bahir Dar City, Ethiopia \\ ${ }^{3}$ Department of Health Education and Promotion, Felege Hiwot Referral Hospital, Bahir Dar City, Ethiopia
}

Email address:

Mulatkassie@yahoo.com (M. Kassie), amanuaragaw@yahoo.com (A. Aragaw), balemayehu95@yahoo.com (A. Belay)

To cite this article:

Mulatu Kassie, Amanu Aragaw, Alemayehu Belay. Assessment of the Quality of Directly Observed Treatment Short-Course of Tuberculosis in Bahir Dar City Administration, North West Ethiopia. Science Journal of Public Health. Special Issue: Health Behavior and Public Health. Vol. 3, No. 1-1, 2015, pp. 6-13. doi: 10.11648/j.sjph.s.2015030101.12

\begin{abstract}
The impacts of tuberculosis is not reduced globally, even though WHO recommends on the growing adherence, the use of DOTS and other interventions in many countries. Providing quality of care for tuberculosis patients is crucial in prevention and control of the disease. The aim of this study is to assess the quality of directly observed Treatment Short Course (DOTS) of Tuberculosis at Government health institutions in Bahir Dar city. Institution based cross-sectional study was conducted from June 30 to August 30/2013 in seven public health institutions in Bahir Dar City Administration. Multistage sampling technique was employed to select health institutions and patients; primary data were collected by interviewing Tuberculosis cases and reviewing their records. The data were coded and entered into Epi Info 3.5.1 and exported in to SPSS version 16 for analysis, and findings at $95 \% \mathrm{CI}$ and $\mathrm{p}$ value of less than 0.05 were reported as statistically significant. Result: The results of this study showed that Input, process and output quality parameters were 50.2\%, 40.2\%, and $53.8 \%$ respectively, with the overall quality of $47.8 \%$. Variables such as location of health institution, patient privacy, and marital status of patients were significantly associate with quality of DOTS. [AOR=2.14, (95\% CI, 1.10-4.15)], [AOR=3.57(95\% CI 1.80-7.07)], ([AOR $=0.24$ (95\% CI 0.08-0.77)] respectively. Conclusion and recommendation: Input, process and output qualities of a program were poor in relative to the $100 \%$ WHO requirement and these would have inevitably decreased the total quality of DOTS. Success of tuberculosis therapy could be ensured through strict adherence to all the elements of DOTS strategy Thus, Bahir Dar City Administrative health office should train laboratory Professionals on $\mathrm{AFB}$, construct waiting room, and to have regular supervision which may improve those problems seen at each level.
\end{abstract}

Keywords: Quality, DOTS, Bahir Dar City Administration, Ethiopia

\section{Introduction}

Tuberculosis (TB) is an infectious disease caused by Mycobacterium tuberculosis, a rod-shaped bacillus called "acid-fast" due to its staining characteristics in laboratory. It typically affects the lungs (PTB) but can affect other parts of the body as well (EPTB). The disease is spread via droplet infection when people with pulmonary TB expel the bacilli while coughing,sneezing, talking, etc. Though it affects peoples of all ages and sexes, poverty malnutrition, overcrowding and more recently HIV/AIDS have been known for decades to make some groups more vulnerable to develop the disease. The TB programme emphasizes the need to increase the access of quality DOTS by expanding its diagnostic and treatment services in line with the increasing number of public and private health facilities. $(1,2)$.

DOTS is internationally recommended public health strategy. It has been started in Ethiopia in 1993 GC with its gradual expansion to all parts of the country. It is the most effective approach available for controlling TB. Its strategy for $\mathrm{TB}$ control consists of five main elements listed as: 
political commitment to sustained TB control, case detection by sputum smear microscopy, standard short-course chemotherapy, a system to ensure regular drug supplies, and a standard recording and reporting system. As it has been recommended by WHO, DOTS strategy is very important to improve the adherence of people to tuberculosis treatment through health workers, family members, or community members and important in Preventing spread of drug resistance, reducing burden of TB among PLHA and general population as well as reducing HIV incidence among TB patients. Patients with tuberculosis (TB) have free access to diagnostic and treatment services in all public health institutions. (3).

Quality which is used by advocates of total quality management is doing the right thing, right, right way. It has different dimensions like technical performance, effectiveness of care, efficiency of service delivery, safety, access to services, interpersonal relations, continuity of services, physical infrastructure and comfort and choices. Quality is also expressed as the degree to which health services for individuals and populations increases the likelihood of desired health outcomes and is consistent with professional knowledge. The ultimate goal of quality assessment in health care program is to assess whether a program possesses the right things (input), is doing the right things (processes) and it leads to the right things (outcome) to happen $(4,5)$.

In spite of the fact that TB is preventable, treatable and curable, it is estimated that one third of the world's population is infected by TB and about 8.4 million people developed active TB and 2.3million of them died from the disease each year which accounted for $2.5 \%$ of the global burden of disease and $25 \%$ of all avoidable infectious cause of death. It continued to be the leading cause of death globally despite the availability of reliable diagnostic approach and availability of effective drugs for over decades. (6).

The emergence of MDR TB in many parts of the world, including Ethiopia, is posing serious threat to the control of TB. Drug-resistant TB is a man-made problem, largely being the consequence of human error as a result of individual or combination of factors related to: management of drug supply, patient management, prescription of chemotherapy (inappropriate treatment, incorrect use of anti-TB drugs, or use of poor quality drugs), patient adherence and Poor infection control practice has been identified as a major contributing factor. More recently the emergence of XDR-TB has added to the complexity of TB care and treatment. There were about 310000 cases of MDR-TB among notified TB patients with pulmonary TB in the world in 2011. At the end of February 2012, a total of 424 cases of MDR-TB patients were enrolled on treatment in Ethiopia. It is estimated that about $9 \%$ of MDR-TB cases had XDR-TB (7).Therefore, this study was intended to assess the quality of care delivered for tuberculosis patients in seven public health facilities in Bahir Dar City Administrative, Amhara regional state since only few studies were undertaken in the country.

\section{Methods}

Institution based cross-sectional study was conducted in Bahir Dar City Administration from June 30 to August 30/2013. It is the capital city of Amhara Regional state, which situated $565 \mathrm{~km}$ from Addis Ababa to the Northwest of Ethiopia. It has 17 kebeles and The City population was 277566 as per the 2007 census. There are one referral Government Hospital, 2 private general hospitals, ten health centres, fourteen private medium and higher clinics.

\subsection{Sampling}

Seven randomly selected public health facilities were included in the study from the total of 11 public health facilities within the Zone that were engaged in both diagnosis and treatment of tuberculosis patients. From each health facilities one health professional who works at TB clinic was included in addition, 260 randomly selected TB cases whose age 15 and above and took treatment for two weeks and above were included in the study.

The sample size was determined using a single population proportion formula assuming p (0.34), since similar studies were conducted previously in Jimma Zone, Oromia regional state(19), Considering 5\% margin of error (d) and confidence level of $95 \%(\mathrm{za} / 2=1.96)$. Based on the above information a sample size was 345 .Then correction formula was used since the total population was less than 10000 ,which was (292) and then sample size was multiplied by a factor of 1.5 to correct the design effect for the multi stage sampling and adding $10 \%$ non response rate, the final sample size was 260

\subsection{Data Collection and Management}

Data was collected using a structured questionnaire and checklist through interview and observation. The questionnaire was adopted from reports and instruments of other published journal articles, from Donabedian's quality assessment model in Health care, and FMOH's-TLCP manual. A questionnaire and checklist were prepared in English and translated into Amharic language and was translated back to English by Linguistic graduates and Health professionals to ensure consistency. The prepared questionnaire was pre-tested prior to the actual data collection in Shimbet health center, Bahir Dar City. Four supervisor and 7 data collectors, who have DOTS training was selected and trained for one day by principal investigator. The data was edited, coded, and entered using Epi data version 3.5.1 and exported to SPSS version 16. Using SPSS version 16, descriptive analysis (Mean, $\pm \mathrm{SD}$, median and percentile for continuous variables and frequencies for categorical variables) was conducted.

Bivarate and multivarate analyses were computed whether there is association between quality of DOTS and selected independent variables, respectively. Factors associated with quality of DOTS at bivariate were identified, and the variables with $P$ value of 0.20 and less 
were taken to multivariate analysis and the model was built with backward elimination. Finally, the $P$-values less than 0.05 were considered statistically significant.

\subsection{Ethical Considerations}

Ethical clearance was obtained from ethical review committee of Bahir Dar University and communicated with regional health bureau before the time of data collection. Letter of permission was obtained from the health bureau and Bahir Dar City Administrative. The confidentiality of information was maintained by excluding personal identifiers and interview privately; data were collected after securing informed consent from every participant.

\section{Results}

\subsection{Socio Demographic Characteristics of TB Patients}

In the current study, from the total 260 study participants, 251 responded to the questionnaire, giving a response rate of $96.5 \%$. Among the study participants, $149 \quad(59.4 \%)$ were in the age group of $15-34$. one hundred forty two $(56.6 \%)$ were male; $128(51 \%)$ were married; $155(61.8 \%)$ were living in urban;220 (87.6\%) were orthodox chritian;117 (46.6\%) were not able to read and write and $100(39.8 \%)$ were farmers by occupation , and $189(75.3 \%)$ of the study participants monthly income was less than or equal to 500.00Birr (Table 1)

Table 1. Socio-demographic and economic characteristics of TB patients in Bahir Dar Administrative Zone, August 2013.

\begin{tabular}{llll}
\hline Variable $n=251$ & Age category & Frequency & Percent \\
\hline & 15-24years & 63 & 25.1 \\
Age & 25-34years & 86 & 34.3 \\
& 35-44years & 58 & 23.1 \\
& $\geq 45$ years & 44 & 17.5 \\
\hline
\end{tabular}

\begin{tabular}{llll}
\hline Variable $\mathrm{n}=251$ & Age category & Frequency & Percent \\
\hline \multirow{3}{*}{ Sex } & Male & 142 & 56.6 \\
& Female & 109 & 43.4 \\
\multirow{4}{*}{ Marital Status } & Single & 94 & 37.5 \\
& Married & 128 & 51.0 \\
& Divorced & 20 & 8.0 \\
& Widowed & 9 & 3.6 \\
Residence & Urban & 155 & 61.8 \\
& Rural & 96 & 38.2 \\
Religion & Orthodox & 220 & 87.6 \\
& Muslim & 23 & 9.2 \\
Educational & Protestant & 8 & 3.2 \\
Status & Not able to read \&write & 117 & 46.6 \\
& Primary & 45 & 17.9 \\
& Secondary & 50 & 19.9 \\
Occupation & College and above & 39 & 15.5 \\
& Government employee & 37 & 14.7 \\
& Private employee & 32 & 12.7 \\
& Farmer & 100 & 39.8 \\
Income & Merchant & 73 & 29.1 \\
& Other & 9 & 3.6 \\
& $\leq 500$ & 189 & 75.3 \\
& 501-1000 & 38 & 15.1 \\
& $\geq 1001$ & 24 & 9.6 \\
\hline
\end{tabular}

\subsection{Structural Attributes Quality of DOTS}

From the total 7 health institutions observed to assess the input, all health institutions have at least one DOTS trained health professionals, laboratory professionals trained on AFB except $\mathrm{ZH}$. All health institutions have the latest version of TB manual and on use it, The latest versions of TB register were available and in use in the five healthy facilities only. Flow charts for TB diagnosis were available and in use in three facilities only. All health institutions have all anti TB drugs, but laboratory reagents were not available in one ZC. Regarding supervision of TB clinics only $2(28.6 \%)$ of them were supervised. (Table 2 )

Table 2. The Structural attributes of quality of DOTS at different health institutions in Bahir Dar city Administration zone, August, 2013.

\begin{tabular}{|c|c|c|c|c|c|c|c|c|}
\hline \multirow{2}{*}{ Variables } & & \multicolumn{7}{|c|}{ Name of health institutions } \\
\hline & & FHRH & BHC & HHC & AHC & TAHC & MHC & ZHC \\
\hline \multirow{5}{*}{ Health professionals working in TB clinic } & MD & 4 & 0 & 0 & 0 & 0 & 0 & 0 \\
\hline & $\mathrm{HO}$ & 0 & 02 & 02 & 02 & 01 & 01 & 01 \\
\hline & Nurse & 10 & 04 & 04 & 03 & 02 & 02 & 01 \\
\hline & Lab. & 4 & 02 & 02 & 02 & 01 & 02 & 0 \\
\hline & Pharm & 0 & 0 & 0 & 0 & 0 & 0 & 0 \\
\hline Trained in dots & & 14 & 6 & 6 & 05 & 03 & 03 & 02 \\
\hline availability \& use TB manual & $\begin{array}{l}\text { Yes } \\
\text { No }\end{array}$ & $\sqrt{ }$ & $\sqrt{ }$ & $\sqrt{ }$ & $\sqrt{ }$ & $\sqrt{ }$ & $\sqrt{ }$ & $\sqrt{ }$ \\
\hline Availability and use of lab manual & $\begin{array}{l}\text { Yes } \\
\text { No }\end{array}$ & $\sqrt{ }$ & $\sqrt{ }$ & $\sqrt{ }$ & $\sqrt{ }$ & $\sqrt{ }$ & $\sqrt{ }$ & $\sqrt{ }$ \\
\hline availability\& use TB register & $\begin{array}{l}\text { Yes } \\
\text { No }\end{array}$ & $\sqrt{ }$ & $\sqrt{ }$ & $\sqrt{ }$ & $\sqrt{ }$ & $\sqrt{ }$ & $\sqrt{ }$ & $\sqrt{ }$ \\
\hline Availability and use Flow chart for TB Dx & $\begin{array}{l}\text { Yes } \\
\text { No }\end{array}$ & $\sqrt{ }$ & $\sqrt{ }$ & $\sqrt{ }$ & $\sqrt{ }$ & $\sqrt{ }$ & $\sqrt{ }$ & $\sqrt{ }$ \\
\hline Weight scale availability and use & $\begin{array}{l}\text { Yes } \\
\text { No }\end{array}$ & $\sqrt{ }$ & $\sqrt{ }$ & $\sqrt{ }$ & $\sqrt{ }$ & $\sqrt{ }$ & $\sqrt{ }$ & $\sqrt{ }$ \\
\hline All anti TB drugs availability and use & $\begin{array}{l}\text { Yes } \\
\text { No }\end{array}$ & $\sqrt{ }$ & $\sqrt{ }$ & $\sqrt{ }$ & $\sqrt{ }$ & $\sqrt{ }$ & $\sqrt{ }$ & $\sqrt{ }$ \\
\hline $\begin{array}{l}\text { All Lab equipments and reagents } \\
\text { Drugs and supplies for TB Received from }\end{array}$ & $\begin{array}{l}\text { Yes } \\
\text { No } \\
\text { ZHB }\end{array}$ & $\sqrt{ }$ & $\sqrt{ }$ & $\sqrt{ }$ & $\sqrt{ }$ & $\sqrt{ }$ & $\sqrt{ }$ & $\sqrt{ }$ \\
\hline
\end{tabular}




\begin{tabular}{|c|c|c|c|c|c|c|c|c|}
\hline \multirow{3}{*}{ Variables } & & \multicolumn{7}{|c|}{ Name of health institutions } \\
\hline & & FHRH & BHC & HHC & AHC & TAHC & MHC & ZHC \\
\hline & RHB & $\sqrt{ }$ & $\sqrt{ }$ & $\sqrt{ }$ & $\sqrt{ }$ & $\sqrt{ }$ & $\sqrt{ }$ & $\sqrt{ }$ \\
\hline Use of FIFO/LIFO & $\begin{array}{l}\text { Yes } \\
\text { No }\end{array}$ & $\sqrt{ }$ & $\sqrt{ }$ & $\sqrt{ }$ & $\sqrt{ }$ & $\sqrt{ }$ & $\sqrt{ }$ & $\sqrt{ }$ \\
\hline Determination of drug need & $\begin{array}{l}\text { Quantification } \\
\text { Guess }\end{array}$ & $\sqrt{ }$ & $\sqrt{ }$ & $\sqrt{ }$ & $\sqrt{ }$ & $\sqrt{ }$ & $\sqrt{ }$ & $\sqrt{ }$ \\
\hline Regular supervision & $\begin{array}{l}\text { Yes } \\
\text { no }\end{array}$ & $\sqrt{ }$ & $\sqrt{ }$ & $\sqrt{ }$ & $\sqrt{ }$ & $\sqrt{ }$ & $\sqrt{ }$ & $\sqrt{ }$ \\
\hline Schedule for supervision & $\begin{array}{l}\text { Yes } \\
\text { No }\end{array}$ & $\sqrt{ }$ & $\sqrt{ }$ & $\sqrt{ }$ & $\sqrt{ }$ & $\sqrt{ }$ & $\sqrt{ }$ & $\sqrt{ }$ \\
\hline
\end{tabular}

\subsection{Process Attributes of Quality of DOTS}

Regarding to provider patient interaction, 251 patients were observed while they were receiving their drugs, and the results showed that $184(73.3 \%)$ patients were greeted by provider. one hundred eighty seven $(74.5 \%)$ patients participated in part of decision making processes of service delivery ; $202(80.5 \%)$ patients were advised how to take drugs,51 $(41.6 \%)$ patients were told when the next follow up will be, $150(59.8 \%)$ were advised on the need to comply with treatment, $52(20.7 \%)$ patients were asked by the provider and 49(19.5\%)asked providers about TB drug treatment conditions. Regarding to the facility level ,169 $(67.3 \%)$ patients were given treatment while providers having tables and chair, almost all patients wait stand to receive treatment and $232(92.4 \%)$ of patients bring water to swallow drug in the TB clinic (Table 3)

Table 3. Provider-patient interaction and pattern of services provision in Bahir Dar City Administrative Zone, August 2013.

\begin{tabular}{llll}
\hline Variable & & Frequency & Percent \\
\hline Patients greeted by health & No & 67 & 26.7 \\
professionals & Yes & 184 & 73.3 \\
Patients participated in decision & No & 64 & 25.5 \\
making & Yes & 187 & 74.5 \\
HW properly explained about how & No & 49 & 19.5 \\
to take drugs & Yes & 202 & 80.5 \\
Health workers advised patients & No & 54 & 21.5 \\
when to return for follow up & Yes & 197 & 78.5 \\
Health worker advised patients on & No & 101 & 40.2 \\
the need to comply with Rx & Yes & 150 & 59.8 \\
Health worker explained patients & No & 70 & 58.4 \\
when follow up AFB done & Yes & 50 & 41.6 \\
Health worker advised patients to & No & 50 & 41.6 \\
bring person with S/S of TB & Yes & 70 & 58.4 \\
Provider ask pt for any concern & No & 199 & 79.3 \\
regarding TB treatment & Yes & 52 & 20.7 \\
Patients who asked health & No & 202 & 80.5 \\
professional for any concerns: & Yes & 49 & 19.5 \\
Availability of water in TB unit & No & 19 & 7.6 \\
Provider having chair and table, & Yes & 232 & 92.4 \\
while treating patients & No & 82 & 32.7 \\
\hline
\end{tabular}

\subsection{Respondents Attribute in TB Control Activities}

According to the exit interviewed of patient this study revealed that $63(25 \%)$ TB patients were incurring during treatment process. $45(17.8 \%)$ of them incurred for transportation; $9(3.6 \%)$ of them incurred for provider and laboratory services and the remaining 9(3.6\%) incurred for food and reception. Main means of transportation of TB patients were walking $182(72.5 \%)$, and vehicles (bus and Bajaj), 69(27.5\%). This study revealed that the minimum and maximum time for walking according to the patient report was 10 and 240 minutes, while the mean time-taken to reach the health institutions from patients home was 50.14 minutes walking with ( $\mathrm{SD} \pm 37.37$ ), and the minimum and maximum waiting times were 1 and 60 minutes respectively. Regarding the mean waiting time of TB patients with health care providers while discussing or taking their drugs was, 14.44 minutes with the $(\mathrm{SD} \pm 9.04)$. No patient has been experienced shortage of drugs (Table 4)

Table 4. Description of respondents attributes in TB control activities in Bahir Dar City Administrative zone, August, 2013

\begin{tabular}{llll}
\hline Variable(n=251 ) & Frequency & Percent \\
\hline Cost incur & No & 188 & 74.9 \\
during treatment & Yes & 63 & 25.1 \\
& Transportation & 45 & 17.8 \\
Cost incurred for & Food and Reception & 9 & 3.6 \\
& Provider fee & 0 & \\
& Lab services & 9 & 3.6 \\
Waiting time & less than 10 minute & 60 & 23.9 \\
& 10-20 minute & 151 & 60.2 \\
Time taken to & 21-60 minutes & 40 & 15.9 \\
reach the HF & 31-60minut & 109 & 43.4 \\
Main Means of & 91 minute and above & 96 & 38.2 \\
transportation & Walking & 142 & 5.6 \\
& Vehicle & 69 & 12.7 \\
\hline
\end{tabular}

\subsection{Patients'Unit TB Registry Record}

Record of TB patients who were in intensive and continuation phases was reviewed. All of them were found to have a registered unit TB registration number, almost all $249(99.2 \%)$ of the patients sex and age were recorded; $248(98.8 \%)$ of patients weight were recorded; more than half patients were classified as EPTB $131(52.2 \%)$, the rest $73(29.1 \%)$ and 47(18.7) were Pulmonary negative and pulmonary positive respectively. Initial diagnostic AFB test was done for 120 (47.8\% patients, of which $47(18.7 \%)$ were positive and 73(29.1\%) was negative for AFB microscopy. The category of most patients 222(88.4\%) were new, $17(6.8 \%)$, and 5(2\%), were transfer in and relapse respectively. The treatment outcome of the patients were $20(8 \%)$ cured, $28(11.2 \%)$, treatment complete, $8(3.2 \%)$, defaulter; $4(1.6 \%)$, and $1(0.4 \%)$ were died and treatment failure respectively. The completion of the information in 
the register were $234(93.2 \%)$ complete and $17(6.8 \%)$ incomplete. (Table 5)

Table 5. Descriptions of patients' unit TB registry record in Bahir Dar City Administrative zone, August 2013

\begin{tabular}{|c|c|c|c|}
\hline Variable $(n=251)$ Category & & Frequency & $\%$ \\
\hline \multirow{2}{*}{ Patients TB unit number } & Recorded & 251 & 100 \\
\hline & Not recorded & 0 & 0 \\
\hline \multirow{2}{*}{ Sex of the patient } & Recorded & 249 & 99.2 \\
\hline & Not recorded & 2 & 0.8 \\
\hline \multirow{2}{*}{ Age of the patient } & Recorded & 249 & 99.2 \\
\hline & Not recorded & 2 & 0.8 \\
\hline \multirow{2}{*}{ Weight of the patient } & Recorded & 248 & 98.8 \\
\hline & Not recorded & 3 & 1.2 \\
\hline \multirow{3}{*}{$\begin{array}{l}\text { Initial diagnostic AFB tests } \\
\text { done }\end{array}$} & Positive & 47 & 18.7 \\
\hline & Negative & 73 & 29.1 \\
\hline & Not recorded & 1 & 0.4 \\
\hline \multirow{4}{*}{ Classification of the patient } & PTB + & 47 & 18.7 \\
\hline & PTB- & 73 & 29.1 \\
\hline & EPTB & 131 & 52.2 \\
\hline & New & 222 & 88.4 \\
\hline \multirow{5}{*}{ Category of patients } & Relapse & 5 & 2.0 \\
\hline & Failure & 1 & .4 \\
\hline & Defaulter & 2 & .8 \\
\hline & transfer in & 17 & 6.8 \\
\hline & Others & 4 & 1.6 \\
\hline \multirow{3}{*}{$\begin{array}{l}\text { Drug and its dose given } \\
\text { during intensive phase }\end{array}$} & Recorded & 237 & 94.4 \\
\hline & Not recorded & 2 & .8 \\
\hline & Positive & 1 & .4 \\
\hline \multirow{3}{*}{$\begin{array}{l}\text { Result of } 2^{\text {nd }} \text { month follow } \\
\text { up AFB microscopy }\end{array}$} & Negative & 34 & 13.5 \\
\hline & Not done & 5 & 2.0 \\
\hline & Unrecorded & 1 & .4 \\
\hline \multirow{3}{*}{$\begin{array}{l}\text { Weight of the pt on the } \\
\text { second month }\end{array}$} & Recorded & 93 & 37.1 \\
\hline & Not recorded & 0 & 100 \\
\hline & Cured & 20 & 8.0 \\
\hline \multirow{4}{*}{$\begin{array}{l}\text { Treatment outcome of the } \\
\text { patients }\end{array}$} & Treatment.com & 28 & 11.2 \\
\hline & Died & 4 & 1.6 \\
\hline & T. Failure & 1 & .4 \\
\hline & Defaulter & 8 & 3.2 \\
\hline \multirow{2}{*}{$\begin{array}{l}\text { Completeness of } \\
\text { information on TB registry }\end{array}$} & Complete & 234 & 93.2 \\
\hline & Incomplete & 17 & 6.8 \\
\hline
\end{tabular}

Table 6. patient's satisfaction level in the given service in Bahir Dar City Administrative Zone, August, 2013

\begin{tabular}{|c|c|c|c|c|}
\hline \multirow{2}{*}{ Variables $\mathbf{N}=251$} & \multicolumn{2}{|c|}{ Satisfied } & \multicolumn{2}{|c|}{ Not satisfied } \\
\hline & NO & $\%$ & NO & $\%$ \\
\hline Satisfaction with adequacy and appropriateness of working hours & 143 & 57 & 108 & 43 \\
\hline Satisfaction with the waiting time & 141 & 56.2 & 110 & 43.8 \\
\hline Satisfaction with the time spent by health worker & 125 & 49.8 & 126 & 50.2 \\
\hline Satisfaction with Cleanliness of waiting area & 111 & 44.2 & 140 & 55.8 \\
\hline Satisfaction the overall comfort of the waiting area & 112 & 44.6 & 139 & 55.4 \\
\hline Satisfaction with the cleanliness of the place where pts received service & 125 & 49.8 & 126 & .50 .2 \\
\hline Satisfaction with the cleanliness of instrument / equipment used by the health professional & 129 & 51.4 & 122 & 48.6 \\
\hline Satisfaction of pts with the respect offered by Health professionals & 201 & 80.1 & 50 & 19.9 \\
\hline Satisfaction with measures taken to assure privacy & 173 & 68.9 & 78 & 31.9 \\
\hline Satisfaction with the completeness of the information given to $\mathrm{pt}$ & 197 & 78.5 & 54 & 21.5 \\
\hline Satisfaction with the effectiveness of the service pts received & 209 & 83.3 & 42 & 16.7 \\
\hline Satisfaction of pts with the service provided & 175 & 69.7 & 76 & 30.3 \\
\hline Overall satisfaction & 135 & 53.8 & 116 & 46.2 \\
\hline
\end{tabular}

\subsection{Logistic Regression (Backward) Analysis between Quality of DOTS and Other Variables}

According to multivariate analysis marital status of TB pts those who were divorced and widowed were found to have 4.14 times quality of DOTS services compared to single clients; [AOR $=0.24$ (95\% CI 0.08-0.77)]. Regarding

\subsection{Patient Satisfaction as an Outcome Indicator for Quality of DOTS}

Satisfaction: Twelve satisfaction questions were used to assess satisfaction of clients in the service they received, cronbach's alpha was tested and the result showed that that its alpha value of 0.781 .The contribution of each item was also analyzed and the value revealed that corrected item total correlation of each item was above the cut of point (0.2)

According to the satisfaction of clients with the different aspects of services provided the finding of this study declared that: $143(57 \%)$ were satisfied; in adequacy and appropriateness of working hours; with respect to waiting time 141(56.2\%) were satisfied; almost half of the respondents $125(49.8 \%$ ), were satisfied by the time spent to them by care providers; Satisfaction with regard to cleanliness and Comfortableness of waiting area were $111(44.2 \%)$ and $112(44.6 \%)$ respectively; satisfaction with cleanliness of TB room and equipments /instruments where TB pts get service were 125 (49.8\%) and 129(51.34\%) respectively. Meanwhile relatively higher study participants, $201(80.1 \%)$ and $173(68.9 \%)$ were satisfied with the respect offered by health professionals and measures taken to assure privacy respectively. (Table 6 ) to the site(location) of health institutions ; those urban health institution were found to give 2.14 times more quality of DOTS service compared with those rural health institutions [AOR=2.14,(95\%CI, 1.10-4.15)]. Considering TB patients privacy those patients who got service as having privacy were found to have 3.57 times quality DOTS service compared with those patients who did not deserve it 
[AOR=3.57(95\% CI 1.80-7.07)] (table 7).

Table 7. logistic regression (backward) analysis between quality of DOTS and other variables, in Bahir Dar City Administrative August 2013

\begin{tabular}{|c|c|c|c|c|c|}
\hline \multirow{2}{*}{ Variable } & \multicolumn{2}{|c|}{ Quality of DOTS } & \multirow{2}{*}{ COR $(95 \%$ CI) } & \multirow{2}{*}{ AOR $(95 \%$ CI $)$} & \multirow{2}{*}{ p-value } \\
\hline & Yes & No & & & \\
\hline \multicolumn{6}{|l|}{ Age } \\
\hline $15-24 y s r$ & 30 & 33 & $1.95(1.15-4.35)$ & $0.86(0.31-2.35)$ & 0.76 \\
\hline $25-34 y r s$ & 49 & 37 & $2.84(1.32-6.09)$ & $1.70(0.71-4.11)$ & 0.24 \\
\hline $35-44 y r s$ & 27 & 31 & $1.87(1.87-1.21$ & $1.51(0.62-3.71)$ & 0.36 \\
\hline$>45 y r{ }^{*}$ & 14 & 30 & & & \\
\hline \multicolumn{6}{|l|}{ Marital status } \\
\hline Single & 56 & 38 & & & \\
\hline Married & 55 & 73 & $0.51(0.30-0.88)$ & $0.72(0.40-1.32)$ & 0.28 \\
\hline Divorced and & 9 & 20 & $0.29(0.10-0.82)$ & $0.24(0.08-0.77)$ & 0.02 \\
\hline \multicolumn{6}{|l|}{ Widowed } \\
\hline \multicolumn{6}{|l|}{ Residence } \\
\hline Urban & 86 & 69 & $2.27(1.35-3.84)$ & $1.081(0.41-2.87)$ & 0.88 \\
\hline Rural & 34 & 62 & & & \\
\hline \multicolumn{6}{|l|}{ Educational Status } \\
\hline Illiterate & 48 & 69 & $0.48(0.23-1.01)$ & $0.78(0.25-2.42)$ & 0.67 \\
\hline Primary & 23 & 22 & $0.73(0.306-1.73)$ & $0.801(0.247-2.595)$ & 0.71 \\
\hline Secondary & 26 & 24 & $0.75(0.32-1.76)$ & $0.65(0.20-2.09)$ & 0.47 \\
\hline College \& above & 23 & 16 & & & \\
\hline \multicolumn{6}{|l|}{ Occupation } \\
\hline Government & 19 & 18 & $0.22(0.040-1.24)$ & $0.23(0.04-1.48)$ & 0.12 \\
\hline Non government & 14 & 18 & $0.17(0.033-0.85)$ & $0.17(0.03-1.06)$ & 0.06 \\
\hline Farmer & 37 & 63 & $0.41(0.080-2.11)$ & $0.29(0.049-1.70)$ & 0.17 \\
\hline Merchant & 43 & 30 & & & \\
\hline \multicolumn{6}{|l|}{ 23Income } \\
\hline$\leq 500$ & 89 & 100 & $055(022-128)$ & $071(0.227-2.20)$ & 0.55 \\
\hline $501-1000$ & 16 & 22 & $\begin{array}{l}0.55(0.22-1.28) \\
044(015-124)\end{array}$ & $053(0163-173)$ & 0.29 \\
\hline $1000 \&$ above & 15 & 9 & $0.44(0.15-1.24)$ & $0.53(0.163-1.73)$ & \\
\hline \multicolumn{6}{|l|}{ Distance } \\
\hline$\leq 30$ minutes & 65 & 44 & & & \\
\hline $30-60$ minutes & 42 & 54 & $\begin{array}{l}3.78(0.47-0.81) \\
1.99(0.83-4.74)\end{array}$ & $\begin{array}{l}1.31(0.42-4.05) \\
1.12(0.36-3.45)\end{array}$ & $\begin{array}{l}0.64 \\
0.85\end{array}$ \\
\hline $61-90$ minutes & 4 & 10 & $\begin{array}{l}1.99(0.83-4.74) \\
102(025-4.11)\end{array}$ & $\begin{array}{l}1.12(0.36-3.45) \\
1.08(0.25-4.75)\end{array}$ & $\begin{array}{l}0.85 \\
0.91\end{array}$ \\
\hline$\geq 90$ minutes & 9 & 23 & $1.02(0.25-4.11)$ & $1.08(0.25-4.15)$ & 0.91 \\
\hline \multicolumn{6}{|c|}{ Health institution site } \\
\hline Urban & 99 & 73 & & & \\
\hline Rural & 21 & 58 & $3.75(2.1-6.71)$ & $2.14(1.10-4.15)$ & $0.025^{*}$ \\
\hline \multicolumn{6}{|c|}{ Pts attend in privacy } \\
\hline No & 69 & 112 & & & \\
\hline Yes & 51 & 18 & $0.22(0.12-0.40)$ & $3.57(1.80-7.07)$ & 0.00 \\
\hline
\end{tabular}

\section{Discussion}

According to this study quality of DOTS was assessed by different quality parameters in structural, process and as an outcome ,thus the overall quality of TB care indicated that $52.2 \%$ of the patients received poor quality of care. The result of our study was better than study in Jima in which $66.0 \%$ of patients received poor quality of care (19). The probable reason could be time gap between the studies. However, study in Egypt was slightly better than ours, in which $49.8 \%$ patients received poor quality of care. (12). Geographical difference and health care delivery system might be the possible reasons for the differences.

With regard to marital status, patients who were divorced and widowed were found to have 4.15 times quality of DOTS services compared to single clients. The possible reason might be due to that they may have their own children to assist in taking their own drugs as well as personal maturity might play its own role.

According to our study structural attribute pertaining to quality assessment showed that all health institutions had trained health professionals in DOTS assigned as full time staff 100\%; all anti TB dugs were available for a minimum stock for 3 months (100\%); but trained laboratory professionals on AFB, and AFB laboratory reagents were not available In $14.3 \%$ of seven health institution included in the study.

However, the study done in Jima revealed that all health institutions providing DOTS service had 100\% trained laboratory professionals on AFB and all the essential anti TB drugs. Another study conducted in Tigray also showed that all health facilities had TB drugs supply without interruption. The discrepancy might be related to the fact that our study included health centres that were recently upgraded from health post to health canter and were in transition period so that all preparations including training of manpower .(17,19) 
According to this study access to the TB clinic was found to be $44.2 \%$. In addition waiting room availability, only one $(14.2 \%)$ health institution had it. The result of this study was not in agreement with the National TLCP standard for TB service access to be in walking distance of $\leq 30$ minutes and requirement of every TB clinic to have waiting room.(8)

Majority $85.7 \%$ of health facilities in this study had equipment and materials required for TB control activity as per the national TLCP standard. But, latest version of TLCP registration book, and weight scale, and TB diagnosis flow charts were not available in $14.3 \%$ of the health facilities. However the study done in Tigray region revealed that all health institutions had standard TB registration book and other necessary materials.(16). The possible reason for this discrepancy might be lack of emphasis by different authorities and concerned bodies in the region as registration books are distributed across all the regions in the country from Federal Ministry of health .The relation between location of health institutions and quality of DOTS was also assessed and the result showed that the odds of quality of DOTS service in urban health institutions were about two folds more likely than rural health facilities. The probable reason for this could be in urban health institutions professionals working in TB clinic might be more stable than professionals in rural area with high turnover striving to live in urban area to take the advantage of living in area of better infrastructure. The other possible reason might also be related to the better infrastructure of urban health institutes.

Provider- Patient interaction had pivotal role in quality of DOTS service. Patient- provider interaction with reference to DOTS service among many includes greeting politely, involving patients in decision making, providing advice and others play important role. Our observational result showed that while $73.3 \%$ of patients were greeted politely by their health care providers, three fourth and six in ten of the patients were participated in parts of decision making and were advised to comply with their treatment respectively. These interactions were inadequate to provide quality services as a witness studies conducted in West Africa, western and Eastern Europe showed that inadequate communications between providers and patients could lead to rejection of public health facilities and acting as a barrier for TB control activities and its quality of services. (13)

According to this study most of the process indicators $(96 \%)$ were registered. However sex, age, initial diagnosis of AFB and weight of patients; $0.8 \%, 0.8 \%, 0.4 \%$ and 1.2 respectively were not recorded. This result was better than the study conducted in the SNNPRS, which showed that Tuberculosis registries were incomplete in many cases where; $26.8 \%$ TB patients were not registered, and all progress records were missing in $5.4 \%$ of patients. Even though the percentage of not recorded is very small in this study, minor errors in patient registration process may have negative impact in quality of patient care. The possible reason for this might be due to lack of adequate follow up of trained health professionals working in TB clinic (14)

In current study, small proportion of patients were classified as pulmonary positive $18.7 \%$, making most of the rest pulmonary negative $29.1 \%$ and EPTB $52.2 \%$; which was opposite to the usual expectations in Ethiopia, where it is expected that; from all new cases of TB, $80 \%$ and $20 \%$ would have been pulmonary positive and extra-pulmonary respectively; and from those cases with pulmonary TB $80 \%$ would be Pulmonary positive and $20 \%$ pulmonary negative (3). This might be due to the current HIV/AIDS pandemic where relatively higher proportion pulmonary negative and EPTB cases are frequently reported.

The finding of this study indicated that there was weak supervision patterns as only $2.9 \%$ of health institutions were supervised in the last six months and the supervision pattern was also unplanned, inconsistent and lack feedback. This was against WHO and national recommendations where they recommend strong supportive supervision as part of program communication and quality improvement. $(6,8)$

Regarding TB patients privacy during TB treatment, patients who got service with privacy were found to had 3.6 times quality DOTS service compared with the counterparts of patients who got service without privacy. The possible reason for this could be most of the patients had TB co-infection with HIV which may need attention for patient privacy. Also, every patient needs a separate place to be advised and counseled for provider initiative HIV counseling and testing.

The majority of respondents, $83.3 \%$, were satisfied in the effectiveness of the treatment provided and $69.7 \%$ were satisfied with the overall services they received but dissatisfaction in cleanliness of waiting area, comfortableness of waiting area, waiting time, and adequacy of working hours, in $55.8 \%, 55.4 \%, 43.8 \%, 43 \%$, respectively. Different literatures showed that these were among the major areas of dissatisfaction which can lead to service rejections by the patients and defaulting, treatment failure and drug resistances. $(17,19)$

\section{Conclusion}

Input and process quality parameters which were the main determinants of output quality of a program were poor in relative to the $100 \%$ requirement of world health organization. Thus, Overall quality DOTS was remained low. Availability of AFB trained laboratory professionals, laboratory reagent, use of registration books, flow chart for TB diagnosis, and availability of waiting room for TB patients and accessibility of service were graded poor in quality. Provider- patient interaction, keeping privacy, and supervision of TB clinic were poor. Additionally there was long waiting time observed which can lead to patients' dissatisfaction and failure to adhere to treatment which in turn can lead to service rejection and program failure. Patients were dissatisfied regarding in cleanliness, comfortableness of waiting area, duration of waiting time, and adequacy of working hours of TB clinic.

It is recommended that: The respective health institutions need to provide periodic refreshments on job trainings and 
develop mechanisms to improve the qualities of staffing. The regional health Bureau, Zonal health office, and health institutions need to facilitate accessibility, and construct TB clinic with standards like having proper waiting room. Regular supervision and follow up of TB programme with proper schedule and feedback need to be practiced by each health institution, zonal health office, and Regional health Bureau as their own concerns. Health professional working in TB clinic should treat TB patients by keeping their own privacy and health professionals working in rural health institutions should be stable and provide quality service

\section{Acknowledgements}

We would like to thank Bahir Dar City Administrative Health Office, the seven District Health Offices of the study area and as well as study health institutions that made us access to all requested

\section{References}

[1] FMOH. Guidelines for clinical and programmatic management of TB, leprosy and TB/HIV in Ethiopia, $5^{\text {th }}$ edition. 2012.

[2] FMOH. Tuberculosis national survey in Ethiopia. $1^{\text {st }}$ edition July 2011; Addis Ababa

[3] FMOH. Manual for Tuberculoses, Leprosy and TB/HIV prevention and control program, $5^{\text {th }}$ edition. 2011.

[4] Edwards D. The Definition of Quality and Approaches to Its Assessment, 2003.

[5] Donabedian A. An introduction to quality assurance in health care. New York: Oxford University Press, Inc. 2003.

[6] World Health organization. WHO 2012 Global Tuberculosis Control report. Geneva: World Health Organization; 2012.

[7] F MH. Guideline for Program and clinical management Of Drug resistant tuberculosis First edition, April 2009.

[8] WHO. Treatment of tuberculosis guideline. $4^{\text {th }}$ edition, 2009.
[9] Marta S. Confronting a hidden disease: Tuberculosis in Roma Community. Roma. www.soros.org/initiatives/health/focus/roma.

[10] Sweta G, Sanjay G, d Dig amber B. Reasons for interruption of anti-tubercular treatment as reported by patients with tuberculosis admitted in a tertiary care institution in India, 2007.

[11] Samuel A, Tomoko K. Factors Contributing to Tuberculosis (TB) Defaulter Rate in New Juaben Municipality in the Eastern Region of Ghana. Journd of the National Institute of Public Health 2010.

[12] Nour El-Din M, Elhoseeny T, and Mohsen A.M. Factors affecting defaulting from DOTS therapy under the national programme of tuberculosis control in Alexandria, Egypt, 2010 .

[13] Lienhardt C. and Rustomjee R. Improving Tuberculosis control: an interdisciplinary approach. The Lancet 2006; 367:949-950

[14] Madebo T. Clinical and operational challenges in the control of TB in Southern Ethiopia. Center for international Health University of Bergan Norway, 2003.

[15] Hill P. C, Stevens W, Hill J. Bah, S. DonkoA. R... Risk factors for defaulting from TB treatment: a prospective cohort study of 301 cases in Gambia. The International Journal of TB and lung diseases. 2003.

[16] Mengiste M, Tesfaye W, Madley R. The quality of TB diagnosis in destrict of Tigray Region of Northern Ethiopia. Ethiopian Journal Health Development Journal. 2005

[17] Mengiste M, James N, John W, Amanuel G, Tassew T... Quality of tuberculosis care and its association with patient adherence to treatment in eight Ethiopian districts, May 2009.

[18] Girma A. Quality Assessment of Directly Observed Treatment Short-Course of Tuberculosis in Afar National Regional State,2007.(Not published)

[19] Taddese G, Challi J, and Fitsum G. Assessment of Quality of Care Delivered for Infectious Pulmonary Tuberculosis Patients in Jimma Zone, 2008. 